\title{
Lenalidomide versus bortezomib maintenance after frontline autologous stem cell transplantation for multiple myeloma
}

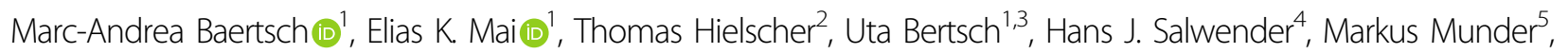

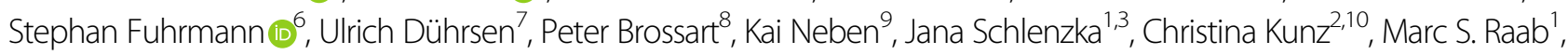
Jens Hillengaß $ß^{1,11}$, Anna Jauch ${ }^{12}$, Anja Seckinger ${ }^{1}$, Dirk Hose ${ }^{1,13}$, Steffen Luntz ${ }^{14}$, Pieter Sonneveld ${ }^{15}$, Henk Lokhorst ${ }^{16}$, Hans Martin ${ }^{17}$, Martin Goerner ${ }^{18}$, Martin Hoffmann ${ }^{19}$, Hans-Walter Lindemann ${ }^{20}$, Helga Bernhard ${ }^{21}$, Igor W. Blau ${ }^{22}$, Christof Scheid $^{23}$, Britta Besemer ${ }^{24}$, Katja C. Weisel $\mathbb{B}^{25}$, Mathias Hänel ${ }^{26}$, Jan Dürig ${ }^{7}$ and Hartmut Goldschmidt $\mathbb{B}^{1,3}$, German-Speaking Myeloma Multicenter Group (GMMG)

\begin{abstract}
Lenalidomide (LEN) maintenance (MT) post autologous stem cell transplantation (ASCT) is standard of care in newly diagnosed multiple myeloma (MM) but has not been compared to other agents in clinical trials. We retrospectively compared bortezomib (BTZ; $n=138)$ or LEN $(n=183)$ MT from two subsequent GMMG phase III trials. All patients received three cycles of BTZ-based triplet induction and post-ASCT MT. BTZ MT $\left(1.3 \mathrm{mg} / \mathrm{m}^{2}\right.$ i.V.) was administered every 2 weeks for 2 years. LEN MT included two consolidation cycles (25 mg p.o., days 1-21 of 28 day cycles) followed by $10-15 \mathrm{mg} /$ day for 2 years. The BTZ cohort more frequently received tandem ASCT (91\% vs. 33\%) due to different tandem ASCT strategies. In the LEN and BTZ cohort, $43 \%$ and $46 \%$ of patients completed 2 years of MT as intended $(p=0.57)$. Progression-free survival (PFS; $H R=0.83, p=0.18$ ) and overall survival (OS; $H R=0.70, p=0.15)$ did not differ significantly with LEN vs. BTZ MT. Patients with $<\mathrm{nCR}$ after first ASCT were assigned tandem ASCT in both trials. In patients with $<n C R$ and tandem ASCT (LEN: $n=54$ vs. BTZ: $n=84$ ), LEN MT significantly improved PFS $(H R=0.61, p=$ 0.04 ) but not $O S(H R=0.46, p=0.09$ ). In conclusion, the significant PFS benefit after eliminating the impact of different tandem ASCT rates supports the current standard of LEN MT after ASCT.
\end{abstract}

\section{Introduction}

High dose chemotherapy (HDCT) followed by autologous stem cell transplantation (ASCT) substantially

Correspondence: Hartmut Goldschmidt (hartmut.goldschmidt@med.uniheidelberg.de)

${ }^{1}$ Department of Internal Medicine V, University Hospital Heidelberg, Heidelberg, Germany

2Division of Biostatistics, German Cancer Research Center (DKFZ), Heidelberg, Germany

Full list of author information is available at the end of the article

These authors contributed equally: Marc-Andrea Baertsch, Elias K. Mai

A list of members with authorship and their affiliations appears at the end of the paper.

All GMMG trial sites involved in the HD4 and MM5 trials are listed in the

Supplementary information. improves PFS in newly diagnosed MM and remains a standard of care for fit patients in the era of novel agents ${ }^{1-4}$. Immunomodulatory drugs (IMiDs), proteasome inhibitors (PIs), monoclonal antibodies and their combinations are now incorporated into the various treatment sequences of MM based on improved PFS and $\mathrm{OS}^{5-8}$. The favorable toxicity profiles of the novel agents have spurred development of continuous treatment regimens which can be administered until disease progression and further improve survival ${ }^{8,9}$.

LEN maintenance treatment (MT) after HDCT/ASCT has become standard of care based on three phase III clinical trials (CALGB $100104^{10}$, GIMEMA RV-MM-PI-209², IFM

\section{(c) The Author(s) 2021}

(c) (i) Open Access This article is licensed under a Creative Commons Attribution 4.0 International License, which permits use, sharing, adaptation, distribution and reproduction cc) in any medium or format, as long as you give appropriate credit to the original author(s) and the source, provide a link to the Creative Commons license, and indicate if changes were made. The images or other third party material in this article are included in the article's Creative Commons license, unless indicated otherwise in a credit line to the material. If material is not included in the article's Creative Commons license and your intended use is not permitted by statutory regulation or exceeds the permitted use, you will need to obtain permission directly from the copyright holder. To view a copy of this license, visit http://creativecommons.org/licenses/by/4.0/. 
2005-02 ${ }^{11}$ ) documenting superior progression-free survival (PFS) and a large meta-analysis $(n=1208)$ revealing an estimated overall survival (OS) benefit of 2.5 years versus observation or placebo ${ }^{8}$. Results from the MRC XI trial recently confirmed the PFS benefit ${ }^{12}$.

However, about $30 \%$ of patients discontinue LEN MT due to a diverse range of treatment emergent adverse events ${ }^{8}$. These include hematotoxicity, general disorders, diarrhea, second primary malignancies (SPM) and others. Many clinical trials have investigated alternative agents for MT ranging from glucocorticoids, interferon alpha and thalidomide to bortezomib (BTZ) and-most recently -ixazomib ${ }^{13}$. While a PFS benefit and in some cases an OS benefit has been reported for these regimens, comparative clinical trials against today's standard of LEN MT have not been published. Several institutions have retrospectively reported their experiences with different agents for MT in clinical practice, but interpretability of these analyses is limited by selection of MT according to baseline patient factors including cytogenetic risk, which makes direct comparison of the different MT agents difficult ${ }^{14,15}$.

BTZ is frequently recommended as an alternative, riskadapted approach for MT in patients with adverse disease characteristics, e.g., by MAYO clinic SMART criteria ${ }^{16}$ and German-speaking Myeloma Multicenter Group (GMMG) standards ${ }^{5,17,18}$. The randomized phase III HOVON-65/GMMG-HD4 trial ${ }^{5,17-19}$ reported amelioration of the poor prognosis associated with deletion $17 \mathrm{p}$ and renal insufficiency with a regimen incorporating BTZ into induction and MT vs. classical chemotherapy induction and thalidomide MT.

Data regarding the benefit of LEN MT in patients with adverse disease characteristics is somewhat conflicting. The meta-analysis by McCarthy et al. ${ }^{8}$ showed only a marginal effect of LEN maintenance versus placebo/ observation on PFS in the high-risk subgroups of patients with elevated LDH, impaired renal function or adverse cytogenetics such as deletion 17p. No OS benefit was observed in these subgroups and additionally in patients with international staging system (ISS) stage III disease. Conversely, in the MRC XI trial ${ }^{20}$ the PFS benefit of LEN maintenance versus placebo was consistent across subgroups including patients with (ultra) high-risk cytogenetics and ISS stage III. OS was significantly improved by LEN MT in the transplant-eligible part of the MRC XI trial and no heterogeneity between cytogenetic risk groups and ISS stages was observed.

To the best of our knowledge no prospective clinical trial has compared BTZ with LEN MT in the post-ASCT setting yet. We therefore sought to exploit the similar designs of the subsequently conducted, multicenter phase III trials GMMG-HD4 ${ }^{5,19}$ and MM5 $5^{21,22}$ to compare BTZ and LEN MT and investigate treatment effects in relevant disease subgroups without the bias of risk-adapted MT choice.

\section{Methods \\ Trials analyzed}

The present analysis retrospectively compared MT regimens in newly-diagnosed, transplant-eligible $\mathrm{MM}$ from two subsequently conducted multicenter phase III trials: GMMG-HD4, the German part of the HOVON-65/ GMMG-HD4 trial (enrollment period: 05/2005-05/2008, EudraCT No. 2004-000944-26), and GMMG-MM5 (enrollment period: 07/2010-11/2013, EudraCT No. 2010-019173-16). Primary endpoints of both trials have been published previously ${ }^{19,21,22}$. For details and study protocols please see the original publications. The trials were conducted in accordance with the European Clinical Trial Directive, the Declaration of Helsinki and local ethics committees. All patients gave written informed consent. All authors had access to the primary clinical trial data.

\section{Study cohorts and treatment}

The analysis included 138 and 183 patients prior to start of maintenance (HD4, study arm B) and consolidation (MM5, study arms A1/A2) therapy, respectively. All patients included in the present analysis received three cycles of a PI-based induction therapy: either PAD/PAd (BTZ/doxorubicin with either high- [HD4 arm B] or lowdose [MM5 arm A1] dexamethasone, $n=138 / 88)^{23}$ or VCD (BTZ/cyclophosphamide/dexamethasone; MM5 arm A2, $n=95)$. In the HD4 trial, patients were intended to receive upfront tandem high dose melphalan $(200 \mathrm{mg} /$ $\left.\mathrm{m}^{2}, \mathrm{HDM}\right)$ and ASCT followed by BTZ MT $\left(1,3 \mathrm{mg} / \mathrm{m}^{2}\right.$ i.v. every 2 weeks) for 2 years (HD4 study arm B). Patients in the MM5 trial received a single HDM/ASCT, and only in case of less than near complete response $(<\mathrm{nCR})$ after first HDM/ASCT a tandem HDM/ASCT was conducted. Thereafter, LEN MT was administered and included two consolidation cycles $(25 \mathrm{mg}$ p.o., days $1-21$, repeated on day 29 , for 2 cycles) followed by 2 years of initially $10 \mathrm{mg} /$ day p.o., which was increased up to $15 \mathrm{mg} /$ day after 3 months in case of good tolerability. Due to the upper age limit of 65 years in the HD4 trial, patients from the MM5 trial older than 65 years were excluded from the present analysis.

\section{Study endpoints and assessments}

All analyses in the present study are exploratory and retrospective, comparing individual patient data from two separate trials. Endpoints were PFS and OS, OS from first relapse/progression, time on MT and toxicities during MT. Subgroup analyses of PFS and OS according to baseline parameters (cytogenetics, ISS, LDH and renal function) and tandem HDM/ASCT in patients with $<\mathrm{nCR}$ after first HDM/ASCT were conducted. 
PFS and OS events were recorded from start of BTZ MT [HD4] or LEN consolidation therapy [MM5]. PFS events included relapse from $\mathrm{CR}$, progressive disease (PD) or death from any cause, whichever occurred first. Patients with PD after induction were allowed to stay on protocol treatment in the absence of new or progressive end organ damage. These patients are excluded for PFS analysis but evaluated for OS. To harmonize follow-up of the two trials, administrative censoring at 60 months was performed.

Eligibility criteria ${ }^{19,22}$, sample processing for fluorescence in-situ hybridization (FISH) ${ }^{17}$ and response assessments according to the International Myeloma Working Group (IMWG) criteria ${ }^{24}$ have been described previously. Near CR (nCR) as a subcategory of very good partial response (VGPR) was defined as the absence of serum and urine M-protein on electrophoresis and/or standard 24-hour urinary measurement with a positive or missing immunofixation status in the serum and/or urine and/or missing bone marrow examination. High-risk (HR) cytogenetics were defined as either deletion del17p13 and/or translocation $t(4 ; 14)$ and/or gain $1 \mathrm{q} 21>$ 3 copies; standard risk (SR) cytogenetics were defined as absence of HR cytogenetics. Adverse events (AEs) were recorded applying the NCI CTCAE criteria (only if $\geq^{\circ} 3$ for the present analysis; version 3.0 [HD4] and 4.0 [MM5]). Infections were retrospectively categorized according to the suspected infectious agent (bacterial, viral, unknown). Serious adverse events (SAE) were recorded independent from the CTCAE grade.

\section{Statistical analyses}

Fisher's exact test and Wilcoxon test were used to compare categorical and continuous parameters between groups. Distribution of PFS and OS times was estimated by the method of Kaplan and Meier. Univariable and multivariable Cox regression was used to estimate the treatment effect. Hazard ratio (HR) gives increase in risk for LEN compared to BTZ, i.e., a HR $>1$ means BTZ is beneficial whereas a $H R<1$ means $L E N$ is beneficial. Likelihood-ratio test of the model with and without interaction term between treatment and risk factor was performed to determine treatment subgroup effects. In multivariable Cox models, missing covariate values were multiply imputed using the mice algorithm ${ }^{25}$. All statistical analyses were done with R 3.5 (www.r-project.org).

\section{Results}

BTZ and LEN cohorts were balanced regarding baseline characteristics at the time of initiation of induction treatment (Table 1). Median age was 57.0 years (31.0-65.0) and 94\% of patients had a WHO performance status of 0/1. Adverse prognostic features were present in $20 \%$ according to ISS (ISS III), in $24 \%$ according to HR cytogenetics, in $17 \%$ according to elevated lactate
Table 1 Baseline characteristics at the time of treatment initiation, i.e., before bortezomib-based induction treatment.

\begin{tabular}{|c|c|c|c|}
\hline & $\begin{array}{l}\text { BTZ cohort } \\
(n=138)\end{array}$ & $\begin{array}{l}\text { LEN cohort } \\
(n=183)\end{array}$ & $p$ \\
\hline Age (median) & $56(50-61)$ & $57(52-61)$ & 0.22 \\
\hline \multicolumn{4}{|l|}{ Sex } \\
\hline Female & $52(38 \%)$ & $80(44 \%)$ & \multirow[t]{2}{*}{0.30} \\
\hline Male & $86(62 \%)$ & $103(56 \%)$ & \\
\hline \multicolumn{4}{|l|}{ WHO PS } \\
\hline 0 & 66 (48\%) & 87 (48\%) & \multirow[t]{3}{*}{1.00} \\
\hline 1 & $64(46 \%)$ & $84(46 \%)$ & \\
\hline$>1$ & $8(6 \%)$ & $11(6 \%)$ & \\
\hline \multicolumn{4}{|l|}{ MM isotype } \\
\hline \multicolumn{4}{|l|}{ Heavy chain } \\
\hline $\lg G$ & 77 (56\%) & 112 (61\%) & \multirow[t]{4}{*}{0.45} \\
\hline $\lg A$ & 35 (25\%) & 34 (19\%) & \\
\hline $\lg D$ & $1(1 \%)$ & $2(1 \%)$ & \\
\hline LCD & $24(17 \%)$ & 35 (19\%) & \\
\hline \multicolumn{4}{|l|}{ Light chain } \\
\hline Kappa & 90 (66\%) & 126 (69\%) & \multirow[t]{2}{*}{0.63} \\
\hline Lambda & 47 (34\%) & 57 (31\%) & \\
\hline \multicolumn{4}{|l|}{ ISS } \\
\hline I & 49 (38\%) & 85 (47\%) & \multirow[t]{3}{*}{0.33} \\
\hline$\|$ & $52(40 \%)$ & 63 (34\%) & \\
\hline III & $28(22 \%)$ & 35 (19\%) & \\
\hline \multicolumn{4}{|l|}{ Cytogenetics } \\
\hline del17p13 & $14(12 \%)$ & $17(10 \%)$ & 0.70 \\
\hline$t(4 ; 14)$ & $14(12 \%)$ & $14(9 \%)$ & 0.43 \\
\hline gain $1 q>3$ copies & $8(7 \%)$ & $13(8 \%)$ & 0.66 \\
\hline$H R^{a}$ & $28(24 \%)$ & $37(24 \%)$ & 1.00 \\
\hline$S R^{b}$ & 89 (76\%) & $116(76 \%)$ & \\
\hline Elevated LDH & $22(17 \%)$ & 32 (18\%) & 0.88 \\
\hline Renal impairment $^{c}$ & $17(12 \%)$ & $13(7 \%)$ & 0.12 \\
\hline
\end{tabular}

BTZ bortezomib, ISS international staging system, $L D H$ lactate dehydrogenase, LCD light chain disease, LEN lenalidomide, MM multiple myeloma, WHO PS world health organization performance status.

${ }^{a} \mathrm{HR}$ (high risk) cytogenetics were defined as presence of del(17p13) and/or $(4 ; 14)$ and/or gain $1 q 21>3$ copies.

${ }^{\mathrm{b}} \mathrm{SR}$ (standard risk) cytogenetics were defined as absence of high risk features. cSerum creatinine $>2 \mathrm{mg} / \mathrm{dl}$. Data are median (interquartile range) or $n(\%)$.

dehydrogenase (LDH) levels and in 9\% according to renal impairment (RI).

The rate of tandem ASCT was significantly lower in the LEN cohort (LEN: $33 \%$ vs. BTZ: $91 \%$; $p<0.001$ ) and the proportion of patients achieving at least nCR after completion of the HDM/ASCT phase (single or tandem) were higher in the LEN cohort (LEN: $52 \%$ vs. BTZ: $39 \%, p=$ 0.02 ) but $\geq$ VGPR did not differ significantly (LEN: $71 \%$ vs. BTZ: $62 \%$;. $p=0.11$; Table 2). The median interval from initiation of induction treatment to initiation of MT was 8.5 months (interquartile range [IQR] $7.7-10.3$ ) and 9.6 months (IQR 8.6-11.0) in the LEN and BTZ cohorts, respectively $(p=0.0001)$. Median time on MT in the LEN cohort was 24.0 months (IQR 10.6-25.9, including two cycles of LEN consolidation) versus 21.5 months (IQR 
Table 2 Response rates prior to start of maintenance therapy.

\begin{tabular}{|c|c|c|c|}
\hline & BTZ cohort $n=134$ & LEN cohort $n=179$ & $p$ \\
\hline \multicolumn{3}{|l|}{ Single categories } & $<0.001$ \\
\hline$C R$ & 41 (31\%) & 38 (21\%) & \\
\hline $\mathrm{nCR}$ & $11(8 \%)$ & $55(31 \%)$ & \\
\hline VGPR & $31(23 \%)$ & $34(19 \%)$ & \\
\hline$P R$ & $50(37 \%)$ & $42(23 \%)$ & \\
\hline MR & $1(1 \%)$ & $9(5 \%)$ & \\
\hline SD & $0(0 \%)$ & $1(1 \%)$ & \\
\hline \multicolumn{4}{|c|}{ Combined categories } \\
\hline $\mathrm{CR} / \mathrm{nCR}$ & $52(39 \%)$ & $93(52 \%)$ & 0.02 \\
\hline$\geq \mathrm{VGPR}$ & $83(62 \%)$ & 127 (71\%) & 0.11 \\
\hline $\mathrm{PR} / \mathrm{MR} / \mathrm{SD}$ & $51(38 \%)$ & $52(29 \%)$ & 0.11 \\
\hline
\end{tabular}

Data are $n(\%)$.

$C R$ complete response, $n C R$ near $C R, V G P R$ very good partial response, $P R$ partial response, $M R$ minimal response, $S D$ stable disease.

12.7-24.1) in the BTZ cohort $(p=0.0017)$. Rates of any documented dose modification (including dose reductions, delays, interruptions and resumptions, and discontinuations) were similar between the two MT strategies (LEN: $141 / 183$ [77\%] vs. 102/138 [74\%]; $p=$ 0.60). The fraction of patients completing two years of MT according to trial protocols was comparable in both cohorts (LEN: 78/183 [43\%] vs. BTZ: 64/138 [46\%]; $p=0.57$ ).

After a median follow up of 49 and 60 months, 104 (57\%) and 99 (65\%) PFS events have occurred in the LEN and BTZ cohorts, respectively. Median PFS from initiation of MT was not significantly different in the LEN and BTZ cohorts (32.7 vs. 25.9 months; HR [LEN vs BTZ] = $0.83,95 \%$ confidence interval $[95 \% \mathrm{CI}]: 0.63-1.09 ; p=$ 0.18; Fig. 1a). Thirty and 37 OS events have occurred. Median OS from initiation of MT was not reached in either cohort; OS was not significantly different between cohorts (HR [LEN vs BTZ] $=0.70,95 \%$ CI: $0.43-1.13$; $p=0.15$; Fig. $1 \mathrm{~b}$ ). OS at 4 years was $84 \%$ and $76 \%$ in the LEN and BTZ cohort, respectively. OS from first relapse did not differ significantly between the two cohorts $(\mathrm{HR}=0.73 ; 95 \%$ CI $0.42-1.24 ; p=0.25$; Supplementary Fig. 1).

Multivariate analyses considering established baseline prognostic factors and response status after completion of the transplant phase (Table 3) were calculated. Effect size of MT (LEN vs. BTZ) on PFS (HR $=0.83 ; p=0.20)$ and OS (HR $0.65 ; p=0.11$ ) were comparable with univariate analyses. Elevated LDH and HR cytogenetics were prognostic for both PFS and OS, while the ISS stages II and III were prognostic for OS.

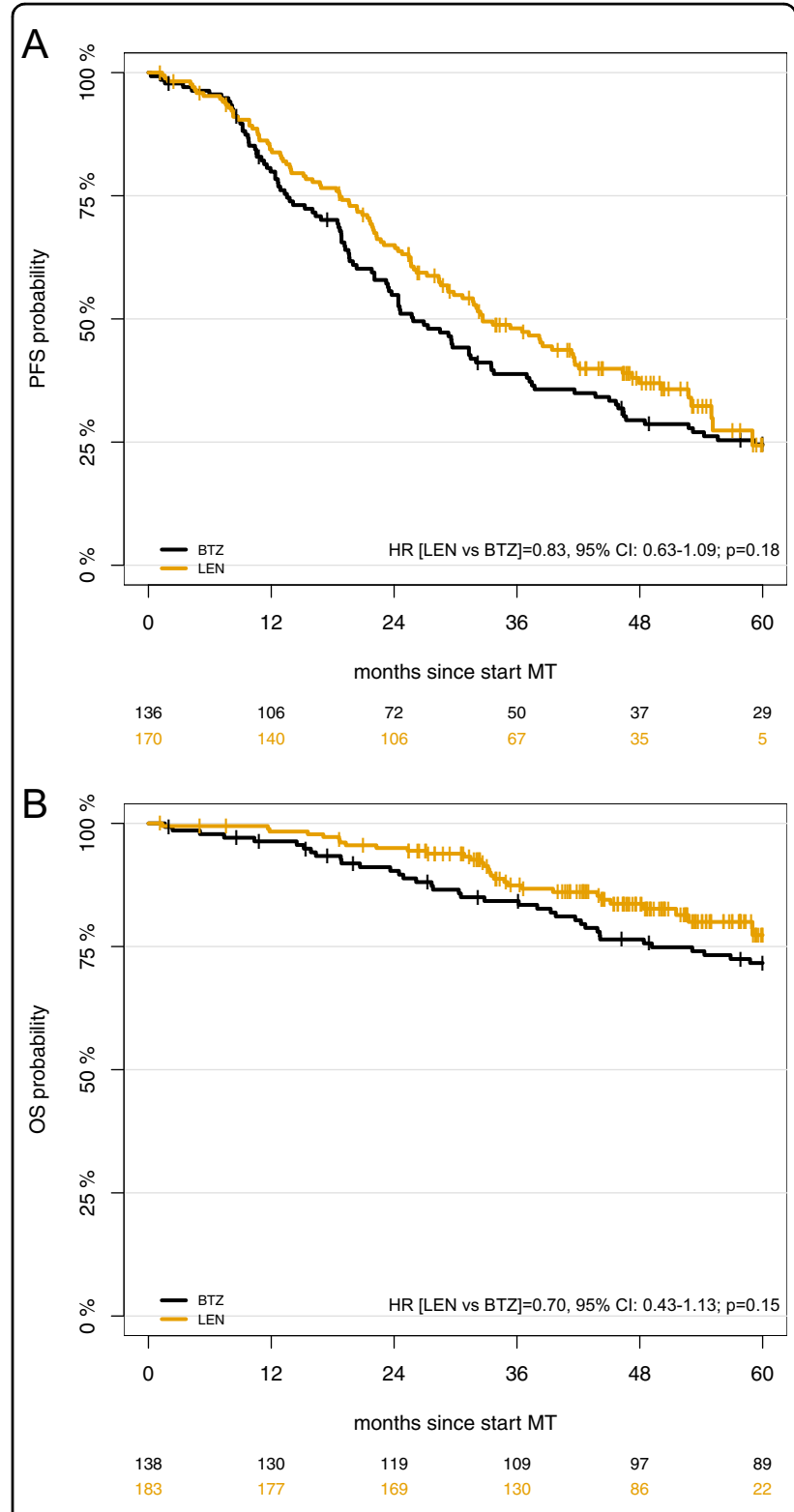

Fig. 1 Progression-free (PFS) and overall survival (OS) in the overall cohorts. Kaplan-Meier curves are shown for (a) PFS and (b) OS. LEN lenalidomide, BTZ bortezomib.

Multivariate subgroup analyses adjusted for response status after completion of the transplant phase/prior to start of $\mathrm{MT}(\mathrm{CR} / \mathrm{nCR}$ vs. $<\mathrm{nCR})$ revealed no significant differential treatment effects for LEN vs. BTZ regarding PFS (Fig. 2). Response-adjusted analyses of OS (Fig. 3) revealed significant differential treatment effects (LEN vs. BTZ) for subgroups according to cytogenetics (SR vs. HR; interaction $\mathrm{p}[\mathrm{i}-\mathrm{p}]=0.049)$, deletion $17 \mathrm{p}(\mathrm{i}-\mathrm{p}=0.018)$, RI (i$\mathrm{p}=0.008)$ and ISS $(\mathrm{i}-\mathrm{p}=0.045)$. No benefit from LEN vs. BTZ MT was observed in patients with deletion17p (HR $=2.56$; 95\% CI: $0.7-9.37 ; p=0.16)$ or RI $(\mathrm{HR}=2.77$; $95 \%$ CI: $0.66-11.66 ; p=0.16)$. Univariate subgroup analyses 
Table 3 Multivariate analysis on PFS and OS.

\begin{tabular}{|c|c|c|c|c|}
\hline & \multicolumn{2}{|l|}{ PFS } & \multicolumn{2}{|l|}{ OS } \\
\hline & HR $(95 \% \mathrm{Cl})$ & $p$ & $\mathrm{HR}(95 \% \mathrm{Cl})$ & $p$ \\
\hline MT (LEN vs. BTZ) & $0.83(0.62-1.10)$ & 0.20 & $0.65(0.39-1.10)$ & 0.11 \\
\hline ISS (II vs. I) & $1.29(0.93-1.79)$ & 0.13 & $2.12(1.13-3.99)$ & 0.02 \\
\hline ISS (III vs. I) & $1.20(0.76-1.92)$ & 0.44 & $2.25(1.05-4.81)$ & 0.04 \\
\hline Cytogenetics (high vs. standard risk) & $1.55(1.10-2.18)$ & 0.01 & $2.10(1.14-3.88)$ & 0.02 \\
\hline LDH (elevated vs. normal) & $1.63(1.12-2.36)$ & 0.01 & $3.31(1.84-5.93)$ & $<0.001$ \\
\hline RI (yes vs. no) & $0.87(0.48-1.57)$ & 0.64 & $0.62(0.26-1.49)$ & 0.29 \\
\hline Age (continuous) & $1.01(0.99-1.03)$ & 0.47 & $1.03(1.00-1.07)$ & 0.08 \\
\hline Response $^{\mathrm{a}}$ (nCR/CR vs. <nCR) & $0.79(0.59-1.06)$ & 0.12 & $0.86(0.51-1.46)$ & 0.58 \\
\hline
\end{tabular}

Cox regression models including PFS: $n=306$ patients, events $n=203$; OS: $n=321$ patients, events $n=67$.

$B T Z$ bortezomib, $C$ confidence interval, $C R$ complete response, $H R$ hazard ratio, ISS international staging system, $L D H$ lactate dehydrogenase, $L E N$ lenalidomide, $M T$ maintenance treatment, $n C R$ near complete response, $R I$ renal impairment (serum creatinine $>2 \mathrm{mg} / \mathrm{dl}$ ).

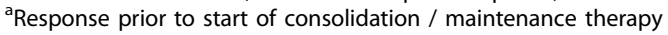

on PFS and OS were similar to response adjusted, multivariate analyses (Supplementary Figs. 2 and 3).

In addition, when patients with RI or deletion $17 \mathrm{p}$ were excluded from the overall analyses, the benefit of LEN vs. BTZ MT became significant in the remaining patients both for PFS (HR $=0.7,95 \%$ CI: $0.5-0.97, p=0.03)$ and OS (HR $=0.46,95 \%$ CI: 0.24-0.87, $p=0.02)$. Differential treatment effects according to cytogenetics (SR vs. HR; i$\mathrm{p}=0.92)$ and ISS $(\mathrm{i}-\mathrm{p}=0.52)$ also disappeared when patients with deletion $17 \mathrm{p}$ or RI were excluded.

To account for differences in tandem ASCT rates, a subgroup analysis was conducted including only patients with suboptimal response $(<\mathrm{nCR})$ after first ASCT and thus receiving a tandem ASCT in both trials. In these $n=$ 54 (LEN cohort) and $n=84$ (BTZ cohort) patients, LEN MT was associated with significantly superior PFS (HR = 0.61, 95\% CI: $0.38-0.98, p=0.04$; Fig. 4a) but not OS $(\mathrm{HR}=0.46,95 \% \mathrm{CI}, 0.19-1.12, p=0.09$; Fig. 4b).

Grade $\geq 3 \mathrm{AE}$ were reported in 123 (67\%) and 79 (57\%) $(p=0.08)$ patients in the LEN and BTZ cohort, respectively (Table 4 ). Grade $\geq 3$ leukopenia/neutropenia was more frequent in the LEN cohort (79/183 [43\%] vs. 3/138 $[2 \%] ; p<0.0001)$. However, this did not translate into an increase in grade $\geq 3$ infections in the LEN cohort $(40 / 183$ [22\%] vs. $42 / 138$ [30\%]; $p=0.09$ ). Grade $\geq 3$ viral infections, especially varicella zoster virus $(\mathrm{VZV})$ reactivations $(14 / 138$ [10\%] vs. $7 / 183$ [4\%]; $p=0.04)$ were more frequent in the BTZ cohort. More grade $\geq 3$ peripheral neuropathies (PNP) were reported in the BTZ cohort (12/ $138[9 \%]$ vs. $3 / 183[2 \%] ; p=0.01$ ).

\section{Discussion}

This is the first comparative report of LEN and BTZ for MT after upfront HDM/ASCT for newly diagnosed MM without the bias of risk-adapted MT choice. While survival with LEN vs. BTZ MT did not differ significantly in the overall cohorts, a significant PFS benefit for LEN MT was observed after eliminating the impact of different tandem ASCT rates.

The strength of our analysis lies in the risk-independent use of both BTZ and LEN MT in the two consecutive phase III clinical trials GMMG-HD4 and -MM5. The similar design of these two multicenter trials with three cycles of BTZ-based triplet induction therapy followed by HDM/ASCT and MT allowed for comparison of the two MT strategies using high-quality, patient-level data. Due to similar eligibility criteria in both trials, baseline characteristics of our BTZ and LEN MT cohorts at the time of treatment initiation including established prognostic factors such as the ISS and HR cytogenetics were well balanced. Furthermore, the two BTZ-based induction regimens used in our patients have been shown to produce equivalent overall response rates, rates of deep responses ( $\geq$ VGPR) and PFS/OS ${ }^{21,23}$.

A limitation of our analysis-apart from its retrospective nature-are the different tandem ASCT policies in the two trials. The general recommendation of tandem ASCT for all patients in the HD4 trial versus responseadapted tandem ASCT for patients in the MM5 trial resulted in a significantly higher rate of tandem ASCT in the HD4/BTZ cohort. The effect of tandem ASCT on survival of patients with $\mathrm{MM}$ is still a matter of debate. In the pre-novel agent era two randomized phase III trials $^{26,27}$ reported a benefit for tandem ASCT while longterm results of the randomized phase III trial GMMG$\mathrm{HD} 2^{28}$ performed in our study group showed no survival differences between single and tandem ASCT after a median follow-up of more than eleven years. In the novel 


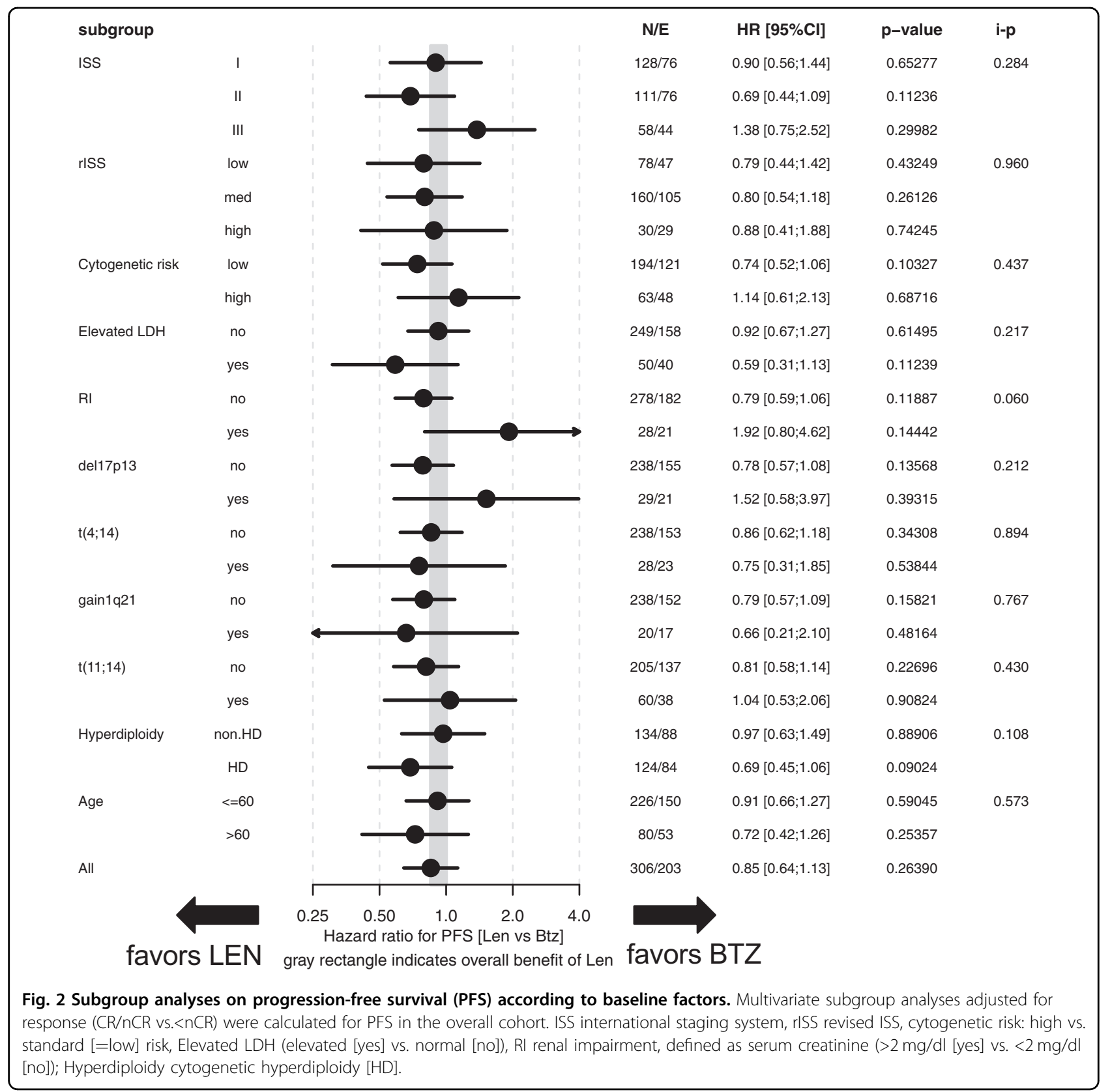

agent era, two phase III clinical trials (EMN02 ${ }^{29}$, STAMINA $^{30}$ ) comparing single vs. tandem ASCT also yielded conflicting results. In studies reporting a benefit, patients with suboptimal response after the first ASCT were amongst those deriving the greatest benefit from a tandem $\mathrm{ASCT}^{26,27}$. These patients, i.e., patients with $<\mathrm{nCR}$ were assigned a tandem transplant in both the LEN and the BTZ cohort. Still, the lower rate of tandem ASCT in the LEN MT cohort may explain the absence of a significant survival benefit for LEN MT in the overall cohorts. In an attempt to eliminate the impact of different tandem ASCT rates, patients who received tandem ASCT for suboptimal response in both trials were compared. In this subgroup the PFS benefit with LEN MT was statistically significant.

An important aspect to consider in interpreting our results is that MM5/LEN MT after BTZ-based induction treatment constitutes a class switch from PI to IMiD, whereas patients in the HD4/BTZ cohort never received an IMiD during their frontline treatment. With the increasingly used BTZ/LEN/dexamethasone (VRD), BTZ/ thalidomide/dexamethasone (VTD) and carfilzomib/ LEN/dexamethasone (KRD) induction and consolidation regimens patients receiving $\mathrm{MT}$ are now frequently pre- 


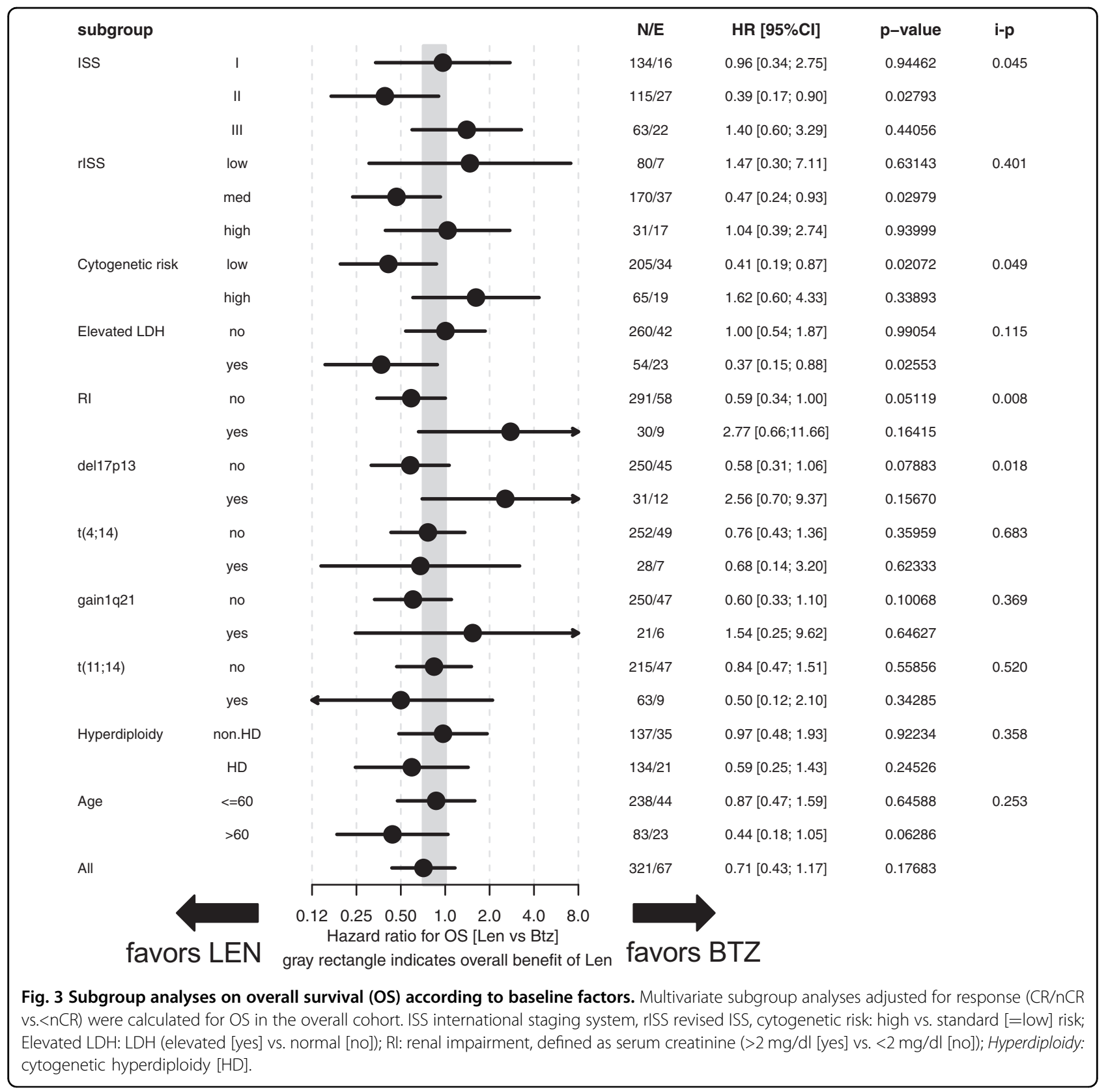

exposed to both a PI and an IMiD which may impact on the relative efficacy of MT with a PI or IMiD. This aspect seems even more relevant since LEN MT in our cohort included two cycles of full dose LEN ( $25 \mathrm{mg} /$ day), followed by 2 years of $10 \mathrm{mg} /$ day (and up to $15 \mathrm{mg} /$ day if tolerated), whereas BTZ was administered at a dose of $1.3 \mathrm{mg} / \mathrm{m}^{2}$ every two weeks for 2 years. Measured against the standard doses of the well-established LEN/dexamethasone (Rd; $25 \mathrm{mg} /$ day, day 1-21 of 28 day cycles) and BTZ/dexamethasone $\left(1.3 \mathrm{mg} / \mathrm{m}^{2}\right.$, day $1,4,8,11$ of 21 day cycles) regimens, the LEN dose administered for MT was considerably higher than the BTZ dose.
Both MT regimens were well tolerated. Expectedly, hematological toxicity of LEN MT was more pronounced. This did not translate into an increase in infections in the LEN cohort. Slightly more infections - mainly viral infections - were observed in the BTZ cohort. In line with the established safety profile of BTZ, significantly more PNP were observed in the BTZ cohort. Intravenous administration of BTZ was standard of care at the time the HD4 trial was conducted, but subcutaneous administration of BTZ is now well established due to less AE, especially PNP ${ }^{31,32}$. Another PI - ixazomib (IXA) - causing less PNP (<1\% grade $\geq 3)$ has been studied for MT. A 


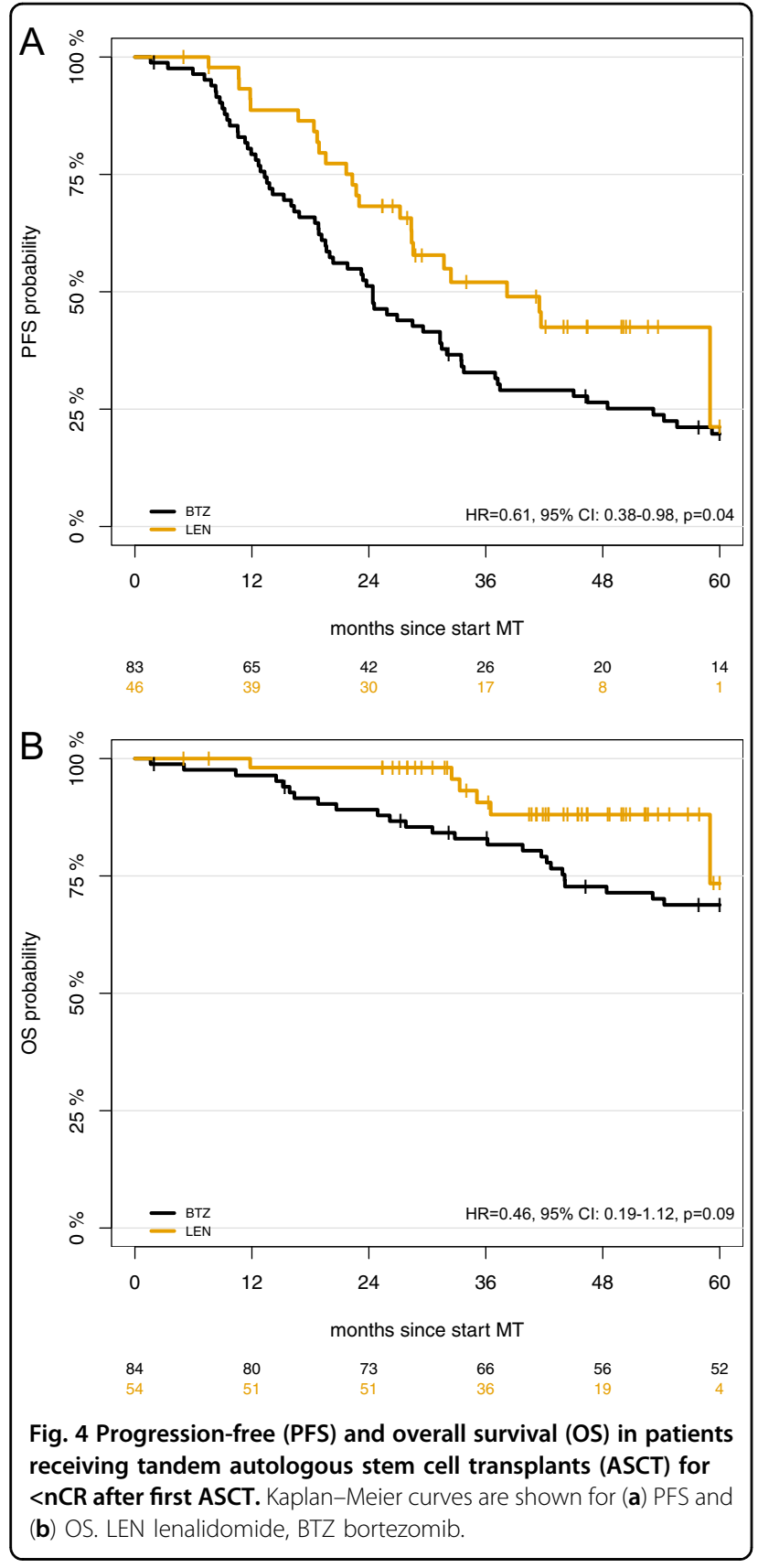

recent phase III clinical trial (TOURMALINE-MM03) demonstrated superior PFS with IXA MT vs. placebo $^{33}$. Oral bioavailability and good tolerability underscore the potential of IXA as MT but no comparative data with other MT agents is currently published.

An important issue with multiple available agents for MT is treatment stratification. The HRs of 0.83 and 0.70 for PFS and OS favoring the LEN vs. BTZ cohort and the significant PFS benefit of LEN MT in patients receiving tandem ASCT for suboptimal response support the current standard of LEN MT. An aim of our analysis was to
Table 4 Grade $\geq 3$ adverse events during maintenance treatment.

\begin{tabular}{lccc}
\hline & $\begin{array}{l}\text { BTZ cohort } \\
\boldsymbol{n}=\mathbf{1 3 8}\end{array}$ & $\begin{array}{l}\text { LEN cohort } \\
\boldsymbol{n}=\mathbf{1 8 3}\end{array}$ & $\boldsymbol{p}$ \\
\hline At least one AE grade $\geq 3$ & $79(57 \%)$ & $123(67 \%)$ & 0.08 \\
Neutropenia/ & $3(2 \%)$ & $79(43 \%)$ & $<0.0001$ \\
Leukocytopenia & & & \\
Anemia & - & $3(2 \%)$ & 0.26 \\
Thrombocytopenia & $6(4 \%)$ & $19(10 \%)$ & 0.06 \\
Infections & $42(30 \%)$ & $40(22 \%)$ & 0.09 \\
$\quad$ Bacterial infections (susp.) & $15(11 \%)$ & $21(12 \%)$ & 1.00 \\
$\quad$ Viral infections (susp.) & $23(17 \%)$ & $14(8 \%)$ & 0.01 \\
VZV & $14(10 \%)$ & $7(4 \%)$ & 0.04 \\
Unknown etiology & $9(7 \%)$ & $11(6 \%)$ & 1.00 \\
Gastrointestinal disorders & $7(5 \%)$ & $7(4 \%)$ & 0.59 \\
Peripheral neuropathy & $12(9 \%)$ & $3(2 \%)$ & 0.01 \\
DVT/PE & $2(1 \%)$ & $4(2 \%)$ & 0.70 \\
Rash & $2(1 \%)$ & $7(4 \%)$ & 0.31 \\
\hline
\end{tabular}

Data are $n$ (\%). Only events $\geq$ CTCAE grade 3 are analysed.

DVT/PE Deep vein thrombosis/pulmonary embolism, VZV Varicella zoster virus, LEN lenalidomide, BTZ bortezomib.

reassess the previously reported benefit of BTZ MT in patients with RI or deletion 17p: The HD4 trial showed marked improvement of PFS and OS with BTZ-based induction and MT versus now obsolete vincristine/doxorubicine/dexamethasone (VAD) induction and thalidomide MT in these high-risk subgroups ${ }^{5,17,18}$. Using the original data from the HD4 trial we did not observe a significant benefit when compared against BTZ-based induction and LEN MT. However, we still found HR in the range of 1.5 to 2.8 in favor of BTZ MT both for PFS and OS pointing towards limited statistical power.

While our results need to be interpreted with caution due to the retrospective nature and the limitations discussed above, they support the current standard use of LEN MT. In patients that cannot tolerate LEN MT, BTZ may be considered as an alternative. Randomized controlled trials comparing different MT agents and combinations are warranted, especially in patients with high-risk features such as deletion $17 \mathrm{p}$ and RI.

\section{Acknowledgements}

We thank the investigators, the study nurses and all the members of the study teams at the participating GMMG trial sites, the teams of the myeloma research laboratory, the FISH laboratory and the central laboratory at the University Hospital Heidelberg, the coordination centers for clinical trials (KKS) in Heidelberg, Köln, Dresden and Leipzig, the pharmacies at the trial sites and, most importantly, the participating patients and their families. The GMMGMM5 trial was supported by grants from Celgene, Janssen-Cilag, Chugai and 
Binding Site. The HOVON-65/GMMG-HD4 trial was supported by the Dutch Cancer Foundation, by the German Federal Ministry of Education and Research, and by a grant from Janssen Cilag. The GMMG also received grants for this trial by Novartis, Amgen, Chugai, Roche, and the Tumorzentrum Heidelberg/Mannheim.

\section{Data sharing and declaration}

Data from the GMMG-HD4 and -MM5 trial is not publicly available. For requests please contact the corresponding author.

\section{Author details}

${ }^{1}$ Department of Internal Medicine V, University Hospital Heidelberg, Heidelberg, Germany. ${ }^{2}$ Division of Biostatistics, German Cancer Research Center (DKFZ), Heidelberg, Germany. ${ }^{3}$ Nationales Centrum für Tumorerkrankungen (NCT) Heidelberg, Heidelberg, Germany. ${ }^{4}$ Asklepios Tumorzentrum Hamburg, AK Altona and AK St. Georg, Hamburg, Germany. ${ }^{5}$ Department of Internal Medicine III, University Medical Center Mainz, Mainz, Germany. ${ }^{6}$ Department of Hematology and Stem Cell Transplantation, Helios Hospital Berlin Buch, Berlin, Germany. ${ }^{7}$ Department of Hematology, University Clinic Essen, Essen, Germany. ${ }^{8}$ University Hospital Bonn, Bonn, Germany. ${ }^{9}$ Department of Hematology and Oncology, Klinikum Baden Baden, Baden Baden, Germany. ${ }^{10}$ Institute of Child Nutrition, Max Rubner Institute, Federal Research Institute of Nutrition and Food, Karlsruhe, Germany. ${ }^{11}$ Roswell Park Comprehensive Cancer Center, Buffalo, NY, USA. ${ }^{12}$ Institute of Human Genetics, University of Heidelberg, Heidelberg, Germany. ${ }^{13}$ Department of Hematology and Immunology, Myeloma Center Brussels, Jette, Belgium. ${ }^{14}$ Coordination Centre for Clinical Trials (KKS) Heidelberg, Heidelberg, Germany. ${ }^{15}$ Department of Hematology, Erasmus MC Cancer Institute, Rotterdam, the Netherlands.

${ }^{16}$ Department of Hematology, VU University Medical Center, Amsterdam, the Netherlands. ${ }^{17}$ Department of Medicine, Hematology/Oncology, GoetheUniversity of Frankfurt, Frankfurt, Germany. ${ }^{18}$ Department of Hematology, Oncology and Palliative Care, Klinikum Bielefeld, Bielefeld, Germany. ${ }^{19}$ Medical Clinic A, Klinikum Ludwigshafen, Ludwigshafen, Germany. ${ }^{20}$ Department of Hematology and Oncology, Katholisches Krankenhaus Hagen, Hagen, Germany. ${ }^{21}$ Internal Medicine V, Klinikum Darmstadt, Darmstadt, Germany. ${ }^{22}$ Medical Clinic, Charité University Medicine Berlin, Berlin, Germany. ${ }^{23}$ Department of Internal Medicine I, University Hospital Köln, Köln, Germany. ${ }^{24}$ Department of Hematology, Oncology and Immunology, University Hospital Tübingen, Tübingen, Germany. ${ }^{25}$ Department of Oncology, Hematology and Bone Marrow Transplantation with Section of Pneumology, University Medical Center Hamburg-Eppendorf, Hamburg, Germany. ${ }^{26}$ Department of Internal Medicine III, Klinikum Chemnitz, Chemnitz, Germany

\section{Author contributions}

$M A B, E K M, H G$ and $T H$ designed the study and analyzed the data. MAB, EKM, UB, JS, HJS, MM, SF, UD, PB, KN, MSR, DH, PS, HL, HM, HWL, IWB, CS, KCW, MH, and JD collected data. MAB, EKM and TH wrote and all co-authors read, discussed, revised and approved the manuscript.

\section{Funding}

Open Access funding enabled and organized by Projekt DEAL.

\section{Conflict of interest}

MAB: Takeda: Consultancy, honoraria; Novartis: Consultancy, Research Funding; Celgene, Amgen, Janssen: Travel grants. EKM: honoraria: Janssen, Celgene, Takeda; consulting or advisory role: Janssen, Celgene, Takeda; research funding: Takeda; travel, accommodations, expenses: Janssen, Takeda, Celgene, Mundipharma. TH: no COI. UB: no COI. HJS: Amgen: Honoraria, Other: Travel or accommodations; Bristol-Myers Squibb: Honoraria, Other: Travel or accommodations; Janssen Cilag: Honoraria, Other: Travel or accommodations; Sanofi: Honoraria, Other: Travel or accommodations; Celgene: Honoraria, Other: Travel or accommodations; AbbVie: Honoraria; Takeda: Honoraria, Other: Travel or accommodations. MMu: honoraria: Janssen, BMS, Takeda, Celgene, Amgen; consulting or advisory role: Janssen, BMS, Takeda, Celgene, Amgen; research funding: BMS; travel, accomodations, expenses: Janssen, BMS, Takeda, Amgen. SF: Advisory board: BMS, Celgene und Amgen. UD: Alexion: Honoraria; Janssen: Honoraria. PB: consulting or advisory role: BMS, AMGEN, Roche, MSD; research funding: BMS; travel, accomodations, expenses: BMS. KN: no COI. JS: no COI. CK: no COI. MSR: honoraria: Celgene, BMS,Novartis, Janssen, Takeda; consulting or advisory role: Celgene, BMS,Novartis, Janssen, Takeda; research funding: Celgene, Novartis, AMGEN; travel, accomodations, expenses: Janssen, BMS, Takeda. JH: Advisory boards: Adaptive, Amgen, BMS, Celgene, GSK, Janssen, Oncotracker. AJ: no COI. AS: no COI. DH: no COI. SL: no COI. PS: SkylineDx: Membership on an entity's Board of Directors or advisory committees; Karyopharm: Research Funding; Amgen: Honoraria, Research Funding; Celgene: Honoraria, Research Funding; Janssen: Honoraria, Research Funding. HL: Janssen: Honoraria, Research Funding; Genmab: Honoraria, Research Funding; Amgen: Honoraria. HM: no COI. MG: no COI. MHo: honoraria: MSD. HWL: no COI. HB: no COI. IWB: research funding: Celgene, BMS, Janssen. CS: honoraria: BMS, Janssen, Celgene, Novartis, Amgen, Takeda; consulting or advisory role: BMS, Janssen, Celgene, Novartis, Amgen, Takeda; speakers bureau: Takeda; research funding: Takeda, Novartis; travel, accomodations, expenses: BMS, Janssen, Celgene, Novartis, Amgen, Takeda. BB: no COI. KCW: honoraria: AMGEN, BMS, Celgene, Novartis, Janssen, Takeda; consulting or advisory role: AMGEN, BMS, Celgene, Juno, Janssen, Adaptive, Sanofi, Takeda; research funding: AMGEN, Celgene, Sanofi, Janssen. MHä: honoraria: Novartis, Amgen, Roche, Takeda; consulting or advisory role: Celgene. JD: consulting or advisory role: Celgene; speakers bureau: Celgene; travel, accomodations, expenses: Celgene. HG: honoraria: Amgen, BMS, Celgene, Chugai, Janssen, Novartis, Takeda; consulting or advisory role: Amgen, BMS, Celgene, Chugai, Janssen, Novartis, Takeda; speakers bureau: Amgen, BMS, Celgene, Janssen, Novartis, Takeda; research funding: Amgen, BMS, Celgene, Chugai, Janssen, Novartis, Takeda; travel, accomodations, expenses: BMS, Celgene, Janssen, Novartis, Takeda.

\section{Publisher's note}

Springer Nature remains neutral with regard to jurisdictional claims in published maps and institutional affiliations.

Supplementary Information accompanies this paper at (https://doi.org/ 10.1038/s41408-020-00390-3).

Received: 16 July 2020 Revised: 29 October 2020 Accepted: 10 November 2020

Published online: 07 January 2021

\section{References}

1. Attal, M. et al. Lenalidomide, bortezomib, and dexamethasone with transplantation for myeloma. N. Engl. J. Med. 376, 1311-1320 (2017).

2. Palumbo, A. et al. Autologous transplantation and maintenance therapy in multiple myeloma. N. Engl. J. Med. 371, 895-905 (2014).

3. Kumar, S. K. et al. Multiple myeloma, version 3.2017, NCCN clinical practice guidelines in oncology. J. Nat. Comp. Cancer Netw. 15, 230-269 (2017).

4. Moreau, P. et al. Multiple myeloma: ESMO clinical practice guidelines for diagnosis, treatment and follow-upt. Annals Oncol. 28, iv52-iv61 (2017).

5. Goldschmidt, $H$. et al. Bortezomib before and after high-dose therapy in myeloma: long-term results from the phase III HOVON-65/GMMG-HD4 trial. Leukemia 32, 383-390 (2018).

6. Moreau, P. et al. VTD is superior to VCD prior to intensive therapy in multiple myeloma: results of the prospective IFM2013-04 trial. Blood 127, 2569-2574 (2016).

7. Moreau, P. et al. Bortezomib, thalidomide, and dexamethasone with or without daratumumab before and after autologous stem-cell transplantation for newly diagnosed multiple myeloma (CASSIOPEIA): a randomised, openlabel, phase 3 study. The Lancet 394, 29-38 (2019).

8. McCarthy, P. L. et al. Lenalidomide maintenance after autologous stem-cell transplantation in newly diagnosed multiple myeloma: a meta-analysis. J. Clin. Oncol. 35, 3279-3289 (2017).

9. Benboubker, L. et al. Lenalidomide and dexamethasone in transplantineligible patients with myeloma. N. Engl. J. Med. 371, 906-917 (2014).

10. McCarthy, P. L. et al. Lenalidomide after stem-cell transplantation for multiple myeloma. N. Engl. J. Med. 366, 1770-1781 (2012).

11. Attal, M. et al. Lenalidomide maintenance after stem-cell transplantation for multiple myeloma. N. Engl. J. Med. 366, 1782-1791 (2012). 
12. Jackson, G. H. et al. Lenalidomide maintenance versus observation for patients with newly diagnosed multiple myeloma (Myeloma XI): a multicentre, openlabel, randomised, phase 3 trial. Lancet Oncol. 20, 57-73 (2019).

13. Vaxman, I. \& Gertz, M. Risk adapted post-transplant maintenance in multiple myeloma. Expert Rev. Hematol. 12, 107-118 (2019).

14. Chakraborty, R. et al. Outcomes of maintenance therapy with lenalidomide or bortezomib in multiple myeloma in the setting of early autologous stem cell transplantation. Leukemia 32, 712-718 (2018).

15. Huang, J. et al. Lenalidomide vs bortezomib maintenance choice postautologous hematopoietic cell transplantation for multiple myeloma. Bone Marrow Transpl. 53, 701-707 (2018).

16. Mikhael, J. R. et al. Management of newly diagnosed symptomatic multiple myeloma: updated mayo stratification of myeloma and riskadapted therapy (mSMART) consensus guidelines 2013. Mayo Clinic Proc. 88, 360-376 (2013)

17. Neben, $\mathrm{K}$. et al. Administration of bortezomib before and after autologous stem cell transplantation improves outcome in multiple myeloma patients with deletion 17p. Blood 119, 940-948 (2012).

18. Scheid, C. et al. Bortezomib before and after autologous stem cell transplantation overcomes the negative prognostic impact of renal impairment in newly diagnosed multiple myeloma: a subgroup analysis from the HOVON65/GMMG-HD4 trial. Haematologica 99, 148-154 (2014).

19. Sonneveld, P. et al. Bortezomib induction and maintenance treatment in patients with newly diagnosed multiple myeloma: results of the randomized phase III HOVON-65/ GMMG-HD4 trial. J. Clin. Oncol. 30, 2946-2955 (2012).

20. Jackson, G. H. et al. Lenalidomide maintenance versus observation for patients with newly diagnosed multiple myeloma (Myeloma XI): a multicentre, openlabel, randomised, phase 3 trial. Lancet Oncol. 20, 57-73 (2019).

21. Mai, E. K. et al. Phase III trial of bortezomib, cyclophosphamide and dexamethasone (VCD) versus bortezomib, doxorubicin and dexamethasone (PAd) in newly diagnosed myeloma. Leukemia 29, 1721-1729 (2015).

22. Goldschmidt, H. et al. Response-adapted lenalidomide maintenance in newly diagnosed myeloma: results from the phase III GMMG-MM5 trial. Leukemia $\mathbf{3 4}$ 1853-1865 (2020).
23. Mai, E. K. et al. Bortezomib-based induction therapy with high or low-dose dexamethasone in newly diagnosed, transplant-eligible multiple myeloma. Leukemia 33, 258-261 (2019).

24. Durie, B. G. M. et al. International uniform response criteria for multiple myeloma. Leukemia 20, 1467-1473 (2006).

25. Buuren S van, Groothuis-Oudshoorn K. Mice: multivariate imputation by chained equations in R. J. Stat. Softw. http://www.jstatsoft.org/v45/i03 (2011).

26. Attal, M. et al. Single versus double autologous stem-cell transplantation for multiple myeloma. N. Engl. J. Med. 349, 2495-2502 (2003).

27. Cavo, M. et al. Prospective, randomized study of single compared with double autologous stem-cell transplantation for multiple myeloma: bologna 96 clinical study. JCO 25, 2434-2441 (2007).

28. Mai, E. K. et al. Single versus tandem high-dose melphalan followed by autologous blood stem cell transplantation in multiple myeloma: long-term results from the phase III GMMG-HD2 trial. Br. J. Haematol. 173, 731-41. (2016).

29. Cavo, M. et al. Autologous haematopoietic stem-cell transplantation versus bortezomib-melphalan-prednisone, with or without bortezomiblenalidomide-dexamethasone consolidation therapy, and lenalidomide maintenance for newly diagnosed multiple myeloma (EMN02/HO95): a multicentre, randomised, open-label, phase 3 study. Lancet Haematol. 7, e456-e468. (2020).

30. Stadtmauer, E. A. et al. Autologous transplantation, consolidation, and maintenance therapy in multiple myeloma: results of the BMT CTN 0702 Trial. JCO 37, 589-597 (2019).

31. Moreau, P. et al. Subcutaneous versus intravenous administration of bortezomib in patients with relapsed multiple myeloma: a randomised, phase 3 non-inferiority study. Lancet Oncol. 12, 431-440 (2011).

32. Merz, M. et al. Subcutaneous versus intravenous bortezomib in two different induction therapies for newly diagnosed multiple myeloma: an interim analysis from the prospective GMMG-MM5 trial. Haematologica 100, 964-969 (2015).

33. Dimopoulos, M. A. et al. Oral ixazomib maintenance following autologous stem cell transplantation (TOURMALINE-MM3): a double-blind, randomised, placebo-controlled phase 3 trial. Lancet 393, 253-264 (2018). 